\title{
The value of the study of natural history in genetic disorders and congenital anomaly syndromes
}

\author{
JUDITH G HALL
}

From the Department of Medical Genetics, University of British Columbia, Vancouver, British Columbia, Canada.

SUMMARY The study of the natural history of genetic disorders and syndromes with congenital anomalies and dysmorphic features is a challenging and often neglected area. There are many reasons to pursue this type of research but it requires special clinical skills and a considerable amount of hard work. Setting up protocols and collecting data is complex and time consuming. Frequently, helpful clues for a particular disorder come from the study of the natural history of other disorders. Older affected subjects and unique cases with unusual features are often most important in unravelling the 'normal' course of a disease or recognising the basic defect. The study of natural history from individual patients and their records is complementary to population or registry based studies because it identifies individual variations and clinical heterogeneity. The understanding of the natural history of a particular disorder is of importance both to the affected person and their family and to the physicians caring for them. It is also useful to the basic researcher trying to determine the pathogenetic mechanism causing the disorder. In many ways, clinical geneticists have learned the art of caring for patients, as well as the challenges of clinical genetics, by becoming apprentices to and studying in depth specific disease entities.

The delineation of syndromes and genetic disorders follows a predictable sequence of stages. First, there is the recognition of a unique association of clinical features. Second, there is clarification of the variability that can be seen, minimal diagnostic criteria, and possibly even establishment of diagnostic tests. Third, there is the elucidation of the natural history and prognosis of the condition. The understanding of the natural history of a particular disorder is of great importance both to the physicians caring for the affected person and to the affected person and their families. Understanding the natural history may lead to prevention, therapy, and even elucidation of the aetiology. However, it is often very difficult to establish the natural history of a particular condition. Because each disorder is relatively rare, there are few cases to observe, and time or ageing are required in order to delineate the natural history. ${ }^{12}$ This paper will emphasise the value of the study of natural history, the need for this form of clinical research, and some of the approaches which can be used.

\section{The definition of natural history}

The natural history of a disorder can be defined as

Received for publication 15 December 1987.

Accepted for publication 5 January 1988. an account of all of the consequences of that disorder, or as the study of the disease process with emphasis on the sequence of events and the effects of time. This includes age of onset, initial complaints, signs, symptoms, laboratory aids in diagnosis, order of onset of complications and manifestations, the effect of the condition on pregnancy, age of death, types and effectiveness of treatments, as well as the modifications that the treatments produce, psychosocial ramifications, and even the impact of ethnic background and environmental factors. $^{2}$

The availability of growth curves and developmental landmarks for the 'normal' affected population allows comparison of an affected person with the 'real' normal. Changes in appearance, variable body proportions, as well as adjustment and productivity, can be defined at all ages. However, there are much less well defined 'norms' available for 'growth and development' in adulthood.

Quantification of physical features is obviously possible. Grading of symptoms and signs and complications can also be established. The social sciences are helping to quantify behaviour, life adjustment, and norms for other psychological and social characteristics which are also part of natural history. With quantification of various aspects of the 
natural history, it becomes possible to make reliable comparisons which can be statistically analysed, and therefore to develop testable hypotheses which can be studied quantitatively.

\section{The importance of the study of natural history}

The study of natural history has many ramifications. It is an essential but often neglected part of clinical genetics. There is a unique and enormous data base that needs to be collected. Knowledge of the natural history of a particular disorder allows improved ability to make a diagnosis, recognition of heterogeneity, and better medical management. It also provides more accurate information for genetic counselling to affected persons and their families with regard to the burden of the disorder, the risks of recurrence, complications, prevention, and therapy for the disorder, and possible reproductive options. Perhaps the most challenging aspect of the study of natural history is the use of this information to try to understand the pathogenetic mechanisms which produce the signs and symptoms of a particular disorder. ${ }^{2}$ We are far from having good natural history information on most genetic disorders and syndromes. In general, single case reports, small clinical series, and anecdotal information are all that is available. A concerted and coordinated effort is needed to define the natural history of genetic disorders and syndromes with congenital anomalies and dysmorphic features.

\section{DIAGNOSIS}

The diagnosis of a particular disorder can be based on clinical features, laboratory data, or a combination of both. For many syndromes and genetic disorders, the diagnosis is made primarily on clinical features since specific diagnostic tests are not available. Therefore, more accurate descriptions of clinical features and the variability that can be seen in a particular condition help to make arriving at a diagnosis an easier process.

With the definition of common clinical features comes the determination of minimal diagnostic criteria, the recognition of additional pathological manifestations, the description of a typical pattern(s) of features, and the clarification of which criteria are most important. Diagnostic systems which differentially weigh particular features can be established, as has been done for neurofibromatosis. ${ }^{34}$

Changes that occur with maturation and age can only be recognised and described by following affected persons over time. Change with ageing is well exemplified by Noonan's syndrome where an evolving phenotype has now been described with a different pattern of features in the infant, young child, older child, and adult. ${ }^{5}$ The changes which occur with aging have important consequences, even in disorders where confirmation of diagnosis is through a laboratory test, as with Down's syndrome. The premature newborn with Down's syndrome and the older person with Down's syndrome are sometimes missed because they do not look like our 'typical image' of childhood Down's syndrome. ${ }^{6}$ However, as the infant ages, the typical features become more obvious and chromosome studies will almost always be requested. In Down's syndrome, functional features such as facial movement (protruding the tongue) and hypotonia may be much more helpful in coming to a diagnosis in the newborn than structural features such as epicanthic folds, Brushfield spots, or simian creases.

In the chondrodysplasias, it is often difficult to make a diagnosis in the newborn period because the bones, which show typical radiographical change for particular disorders, have not yet ossified. The pattern of bony change evolves over time and at a later age may allow a particular diagnosis, often on the basis of bone shape and the presence of abnormal growth centres. Again, in adults with chondrodysplasias, it may become difficult to make a specific diagnosis if it has not been established from childhood $x$ rays, because once the epiphyses are fused the bony changes may be much less

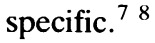

Thus, the definition of the natural history of a disorder improves the ability of the clinician to make a diagnosis at all ages. If accurate measurements and functional studies are available at various ages, it may become possible to make diagnoses on the basis of a pattern of measurements and quantified findings, even though the usual 'gestalt' of facial features is not present. ${ }^{10}$

RECOGNITION OF HETEROGENEITY AND VARIABILITY OF EXPRESSION

Since many syndromes and genetic disorders are still diagnosed on a clinical basis, better definition of the natural history of a particular condition allows the recognition of heterogeneity on the basis of clinical and physical features, on the basis of different types and times of onset of complications, or on the basis of different inheritance patterns. One of the principles of clinical genetics is that similar or identical phenotypes may have very different aetiological bases. In fact, one of the tasks of clinical geneticists over the last two decades has been recognising and delineating heterogeneity. The term, clinical heterogeneity, has been used loosely to encompass nongenetic and genetic heterogeneity, non-allelic and allelic heterogeneity, and even (which has led to confusion) to describe the variability in expression 
of a single gene. Distinctions between these various types of heterogeneity are important.

Non-genetic heterogeneity obviously means that the difference between phenotypically similar subgroups does not occur on a known genetic basis (for example, many causes of intrauterine growth retardation are non-genetic). Genetic heterogeneity can be non-allelic, in which case there are two or more different gene loci responsible for the same or similar phenotypes (as with autosomal dominant Charcot-Marie-Tooth disease where two types were first separated by different linkage ${ }^{11}$ ), or allelic, in which case there are two or more different alleles at the same locus responsible for the same or similar phenotypes (as with the multiple mutants of the $\beta$ globin gene which produce thalassaemia ${ }^{12}$ ). Different alleles at the same locus can also produce surprisingly different phenotypes depending on the type of mutation and the part of the gene affected (as seen with Becker and Duchenne muscular dystrophies ${ }^{13}$ ). However, within a specific family, when the same mutant allele is present, quite different manifestations of a disorder may be seen in different family members. Traditionally, this spectrum of severity is described as 'variability of expression'. Recently, this phenomenon has sometimes been described as 'clinical heterogeneity within the family'. Although heterogeneity as defined by Webster's dictionary does mean "consisting of dissimilar or diverse constituents" ${ }^{14}$ when used in this context its use leads to confusion with genetic heterogeneity and should be avoided.

The possible mechanisms producing variability of expression are of great interest. The comparison of intrafamilial variability with interfamilial variability helps to define some of these processes which include: (1) epistasis or the interaction with nonallelic genes (for example, the interaction of various hyperlipidaemia genes to produce raised serum cholesterol, ${ }^{15}$ (2) environmental modulation of genes, ${ }^{16}$ and (3) regulation of transcription by sites outside the gene. ${ }^{17}$ Furthermore, the difference in manifestations between different ethnic groups may allow better definition of these non-allelic factors.

Clinical heterogeneity becomes obvious to the clinician who studies almost any disorder. The challenge is to determine whether it is genetic or non-genetic, allelic or non-allelic, and whether mechanisms can be defined for the variability of expression seen within a given family.

Genetic heterogeneity has been particularly obvious within the chondrodysplasias. Not very many years ago, there were thought to be only a few dwarfing conditions, but with better clinical information, well over a hundred specific entities are now recognised. ${ }^{18}$ Usually, heterogeneity is first suspected clinically and may later be confirmed by laboratory studies. As biochemical and histological methods are applied to a specific chondrodystrophy, subgroupings may become obvious and reclassification may become necessary. Osteogenesis imperfecta is an excellent example. Sillence's clinical classification ${ }^{19}$ of osteogenesis imperfecta allowed subtypes to be distinguished which then became the basis for applying different biochemical and DNA techniques, looking for the basic defect in collagen. Now that specific collagen defects are being determined, classification of osteogenesis imperfecta may more usefully be based on the domain of the collagen molecule involved and the type of DNA mutation (that is, deletion, nonsense, missense, etc) than on specific clinical features. The work on osteogenesis imperfecta has made it clear as well that the particular diagnostic techniques need to be applied to each subgroup (that is, a person or family from each subgroup should be studied), since marked differences may be observed that would have been missed if only applied to a few patients.

Spranger ${ }^{7}$ has recently suggested that some chondrodystrophies can be categorised into 'families' because of similar features and that these patterns of features reflect a similar pathogenetic mechanism. The preliminary finding of linkage for both Stickler's syndrome and Kniest's syndrome ${ }^{20}$ (both of which were part of one such suggested family) to the type II collagen gene (COL2A1) seems to confirm this approach.

Other work on Duchenne and Becker muscular dystrophies ${ }^{13}$ indicates that each of the two conditions which were thought on clinical grounds to be distinct disorders are actually allelic. It may be that as the years pass, we will often be 'lumping' together disorders which have been 'split' on clinical grounds. ${ }^{21}$

It is sometimes possible within a large group of patients who have a particular disorder to recognise a striking subcategory on the basis of clinical features. This was possible, for instance, in arthrogryposis because patients with amyoplasia ${ }^{22}$ could be distinguished on the basis of their very specific clinical features at birth.

Laboratory tests may be the clue by which subgroups are recognised. For instance, in the case of Prader-Willi syndrome, once a chromosomal abnormality was found in some patients, the previously described patients could be separated into two groups according to whether they have a visible chromosome abnormality or not. Subsequently, differences in the clinical features in the two groups have been defined. ${ }^{23}$

Distinct inheritance patterns, the inclusion or exclusion by linkage, the finding of ethnic differ- 
ences, the observation of different growth patterns, the presence of a particular complication, and the presence or absence of deterioration can all be features which allow the recognition of heterogeneity. The observation of clinical or laboratory heterogeneity encourages the researcher to find explanations for the subgroupings, which may then result in the recognition of different pathogenetic processes.

\section{IMPROVED MANAGEMENT}

Studying the natural history of specific disorders allows one to define and predict specific complications. This has implications for both prognosis and management and since other persons with the condition may be at risk for the observed complications, their health care must be monitored accordingly. Consequently, the 'standard' or 'norm' expected for the 'average' affected patient must be established (that is, the average age of diagnosis, ${ }^{24}$ the age of onset of the disease or particular complication, ${ }^{25}$ the growth curve for the specific condition or syndrome, ${ }^{26}$ life expectancy, ${ }^{27} 28$ etc) in order to judge the effects of a particular type of therapy or preventive measure. The growth curves of various forms of chrondrodysplasia ${ }^{26}{ }^{29}$ and Turner's syndrome ${ }^{30}$ have been established. Now that potentially growth promoting hormonal therapies have become available, ${ }^{31}$ the effectiveness of therapy can be judged against the established 'norms' for the condition.

In a patient with a deletion of $13 \mathrm{q}$, retinoblastoma is likely to occur. ${ }^{31} \mathrm{~A}$ child with a chromosomal deletion in that area must be carefully monitored to allow early diagnosis and therapy. The child with retinoblastoma is also at risk of developing osteosarcomas at a later time. Obviously again, monitoring allows early and therefore probably more successful therapy. Inheritance of the myotonic dystrophy gene from an affected mother puts the child at an increased risk of having more severe disease. Therefore, when an affected woman is pregnant, care and consideration must be given to this potential complication. When the father is the affected parent, severe neonatal myotonic dystrophy is unlikely to occur. ${ }^{32}$ Recently, studies of the eyes of patients with Gardner's syndrome have suggested that most families show pigmentary changes in the retina which can be observed in early childhood allowing recognition of carriers long before GI abnormalities are found. ${ }^{33}$

The awareness of potential complications allows more appropriate management of the patient involved, as well as the development of the most effective therapy. In addition, the effect of various therapies can be quantified, compared, and measured against the untreated natural history. For instance, in the case of achondroplasia, spinal stenosis is a known complication in $30 \%$ of adults. Improved diagnostic and therapeutic treatment of that spinal stenosis has been possible by prospective screening and the development of more appropriate surgical procedures. ${ }^{34}$ In arthrogryposis (multiple congenital contractures), physical therapy is usually the treatment of choice for the contractures and, in general, aggressive physical therapy is advocated for children with arthrogryposis during at least the first six months of life. ${ }^{35}$ Children with diastrophic dysplasia are an exception to this because they not only have multiple congenital contractures, but also have a chondrodysplasia. Because their cartilage is not normal, physical therapy can actually aggravate their contractures leading to bony fusions between phalanges. $^{2}$

Unexpected complications, such as developing malignant hyperthermia with surgery in adults with osteogenesis imperfecta and in one type of arthrogryposis, ${ }^{36}$ suggest a new subcategory of those disorders and also allow physicians to improve the precautions taken in all persons with those disorders at the time of surgery.

Management can sometimes be aided by analogies made with other similar conditions. For instance, work with patients with Morquio's syndrome, and subsequently other forms of spondyloepiphyseal dysplasia, suggested that all patients with any kind of vertebral dysplasia may be at risk for odontoid hypoplasia and subsequent $\mathrm{C} 1$ to $\mathrm{C} 2$ dislocation. ${ }^{8}$ Thus, specific and related complications can be predicted and the general care and monitoring of the patient can be improved by knowledge of natural history.

IMPROVED GENETIC COUNSELLING

The study of natural history and the definition of the particular problems seen in a disorder allow much more accurate information to be provided to patients and families concerning the spectrum of severity and the potential burden of a particular disease.

Knowledge of the specific risk that a condition will recur in family members is very important to them for their own reproductive planning. Fairly accurate empirical predictions can be made for most conditions and prenatal diagnosis is becoming available for an ever increasing number of disorders. Inherent in the diagnosis of most genetic disease or malformation syndromes is a specific recurrence risk for children of the proband and other members of the family. Achondroplasia is always an autosomal dominant trait ${ }^{37}$; by contrast, amyoplasia is always sporadic. $^{22}$ 
Reproductive decisions, however, are based not only on the magnitude of the recurrence risk but also on the anticipated burden of a disorder. Knowledge about the natural history of a disorder is essential for the family to be able to come to an understanding of the burden. For instance, the parents of an anencephalic infant who dies at birth are at risk in the next pregnancy for having a child with spina bifida who would survive and could be handicapped to varying degrees. To provide the family with adequate information in rare conditions, case reports may be scoured, known patients with the same disorder may be contacted, and analogies may be made with similar disorders. However, better studies are still needed for almost every disorder in order to understand the spectrum of severity and the potential burden to the families.

\section{DEFINING THE BASIC DEFECT}

The description of the natural history of a disorder often provides hints about where to look for the basic defect. The time of onset, the pattern of organ system involvement, the distinction of primary versus secondary and tertiary effects, the types of abnormal cell differentiation, the patterns of growth, the presence of static versus progressive changes, and the mode of inheritance all give clues as to the kind of pathogenetic process at work. It is important to keep in mind classes of pathogenetic mechanisms while describing the natural history of a particular disorder, in order to be sure that appropriate questions have been asked. When? What? How? Why? need to be answered. Temporal, spatial, and functional relationships as well as the types of pathological processes need to be established so that testable hypotheses can be developed.

The time of onset (that is, embryo, fetus, newborn, childhood, adolescent, or adult) of a particular abnormality, as well as the age at which it is recognised, provide important clues to the type of process. Is there a problem in shifting from embryonic to fetal proteins (as with embryonic to fetal haemoglobin, or a failure to make normal fetal skin, as in the constrictive dermopathy $\left.{ }^{38}\right)$ ? Or is the problem primarily during childhood (as is seen from examining the growth velocity curve of achondroplasia $\left.{ }^{26}\right)$ ? Certain patterns of structural anomalies in different organ systems can be recognised to have occurred at a particular time in embryonic development, as in thalidomide embryopathy, ${ }^{39}$ or as a failure to go through a normal process, such as delayed puberty. There are 'critical' points in development that provide thresholds which must be crossed (for example, did the palate completely fuse?) and 'benchmarks' (for example, if the neural tube failed to close, it will have done so by day 28 of embryonic development) that help to date a 'failed' or abnormal process.

The pattern of tissue and organ system involvement provides clues about common pathways and enzyme systems, embryonic origins, and spatial relationships. Some abnormalities involve only one tissue type (such as most of the chondrodysplasias); others involve specific body areas (such as those supplied by an artery or nerve) or specific tissues which have derived from a developmental region (such as a branchial arch) or which come from a common embryonic origin (such as neural crest). During the course of embryonic and fetal development, the spatial relationships of various structures change. The thyroid migrates down into the neck, but it can 'get caught' under the tongue. The spleen and gonad migrate past each other and very rarely fuse to each other at that time. The pattern of tissue proteins, enzyme pathways, and responsiveness to various growth factors changes during development, but finding predictable patterns of involvement provides clues to the basic mechanism. The peroxisomal disorders are inborn errors of metabolism which also produce inborn errors of morphogenesis in a predictable way; any combination of malmigration of neurones of the central nervous system, retinal dysplasia, stippled epiphyses, cystic dysplastic kidneys, or deafness could suggest a peroxisomal disorder. ${ }^{40}$

Normally during development structures begin to function. In order to develop fully in utero 'use' is necessary. Many secondary and even tertiary effects may be seen. When the fetus does not move normally, the Pena-Shokeir phenotype (fetal akinesia sequence) may be produced. Pulmonary hypoplasia, congenital contractures, intrauterine growth retardation, small jaw, and short umbilical cord occur secondary to the lack of movement. ${ }^{41}$ The challenge in trying to define basic defects is to determine the primary process, which in this case may be, for instance, either an abnormal central nervous system or abnormal muscle tissue, which then secondarily leads to decreased movement, and thirdly to the clinical features we observe.

Recognition that the primary problem is one of cell differentiation (both failure to occur on schedule and occurring too early in development) or regulation of growth (both over- and undergrowth of tissues or organs) allows specific pathways to be pursued. Disease states can occur not only from abnormal differentiation, but also from abnormal growth patterns once differentiation has occurred (that is, both abnormal over- or undergrowth can produce disease).

The determination of whether a disorder is static or progressive distinguishes ongoing processes from 
abnormalities that occurred during embryogenesis or fetal development and have not undergone further change. Some disorders, such as thrombocytopenia and absent radius, have both static (absence of radius) and ongoing (leukaemoid reactions and thrombocytopenia) disturbances. Others, like Coffin-Lowry syndrome, appear to be slowly progressive, suggesting a metabolic degenerative disturbance.

The pattern of inheritance provides special clues to pathogenetic mechanisms, such as whether the disorder represents a deficiency or an excess, and whether the gene product is defective or whether its regulation is the source of the abnormality. For instance, persons likely to be homozygous for the Huntington's disease gene are no worse clinically than heterozygotes, ${ }^{42}$ suggesting that the abnormal gene is unlikely to produce a deficiency, but rather must have some other pathogenetic mechanism. Sporadic diseases with patchy involvement, such as McCune-Albright syndrome, are likely to represent mosaicism for a new mutation which would be lethal if present in all cells. ${ }^{43}$

The study of natural history is extremely important for pointing the basic scientist toward appropriate areas of research. In addition, the proposed pathogenetic mechanism must explain the feature observed. For instance, the molecular defect in sickle cell anaemia can explain why sickle cell crises occur and why there is increased susceptibility to certain kinds of infections in the affected homozygote. $^{44}$

RESEARCH CONCERNING NATURAL HISTORY Probably the reason that little is known about the natural history of most genetic conditions and syndromes with congenital anomalies and dysmorphic features is that each is relatively rare and it takes an experienced clinician to be able to set up and conduct a study of natural history. Most genetic clinics see patients only once or twice, but many visits over many years with carefully detailed records may be required to establish natural history. Patients with genetic disorders may often never even be seen in genetics clinics. Many syndromes have been recognised for less than 20 years and since it takes many years to accumulate natural history data, there may not have been time to establish a database. In the past, clinical research on the study of natural history has not been well supported by research funding agencies, nor has it been given adequate academic recognition. It requires a different approach from that of classical laboratory research studies or clinical care research studies, but natural history information is clearly needed.
SPECIAL RESOURCES

Collaborative studies are often required for natural history studies. Genetic disorders are relatively rare, so no one physician or centre usually has sufficient affected or at risk patients to be able to set up a study of natural history. Affected persons may be followed in a variety of different clinics to care for specific complications (cardiac, orthopaedic, ENT, etc). Even for relatively common disorders, such as neurofibromatosis, tuberous sclerosis, and Huntington's disease, collaborative studies are needed in order to have sufficient numbers of patients to achieve statistically significant results when testing hypotheses.

Working together with specific disease group or lay group organisations is often one of the best sources of patient material. ${ }^{45}{ }^{46}$ Most affected persons or parents of affected children are very concerned about the particular disorder and are usually willing to cooperate with investigations. These families are highly motivated to have the natural history and the complications of the disorder identified and defined. In addition, parents who deal with the condition on a daily basis often make observations that can lead to a better understanding of the natural history and the basic mechanisms. Examples of patient lay groups which have worked hard to contribute to an understanding of the natural history of particular disorders include Little People of America, the Williams Syndrome Association, the Prader-Willi Association, the Huntington Disease Society, and the Turner Syndrome Association. ${ }^{46}$

Special clinics for specific disorders may serve the same purpose since they allow a number of patients to come together at one time and facilitate the making of comparisons. Usually, special clinics involve a variety of specialists who come together, bringing their own areas of expertise. Because they have an interest in the specific disorder, they will probably have acquired very useful knowledge about it. In addition, special clinics often establish protocols to collect data in an organised way and to define the response to particular types of therapy. Such clinics require excellent organisation, designated clinic coordinators, and usually additional funding. Examples include clinics for short stature, neurofibromatosis, arthrogryposis, and genetic dermatological disorders.

Older adults with a particular disorder can often be a very valuable source of information about natural history. These persons will have lived a relatively long time and therefore have developed many of the complications which are likely to occur. It is only by following patients to a relatively old age that it is possible to determine fully the natural 


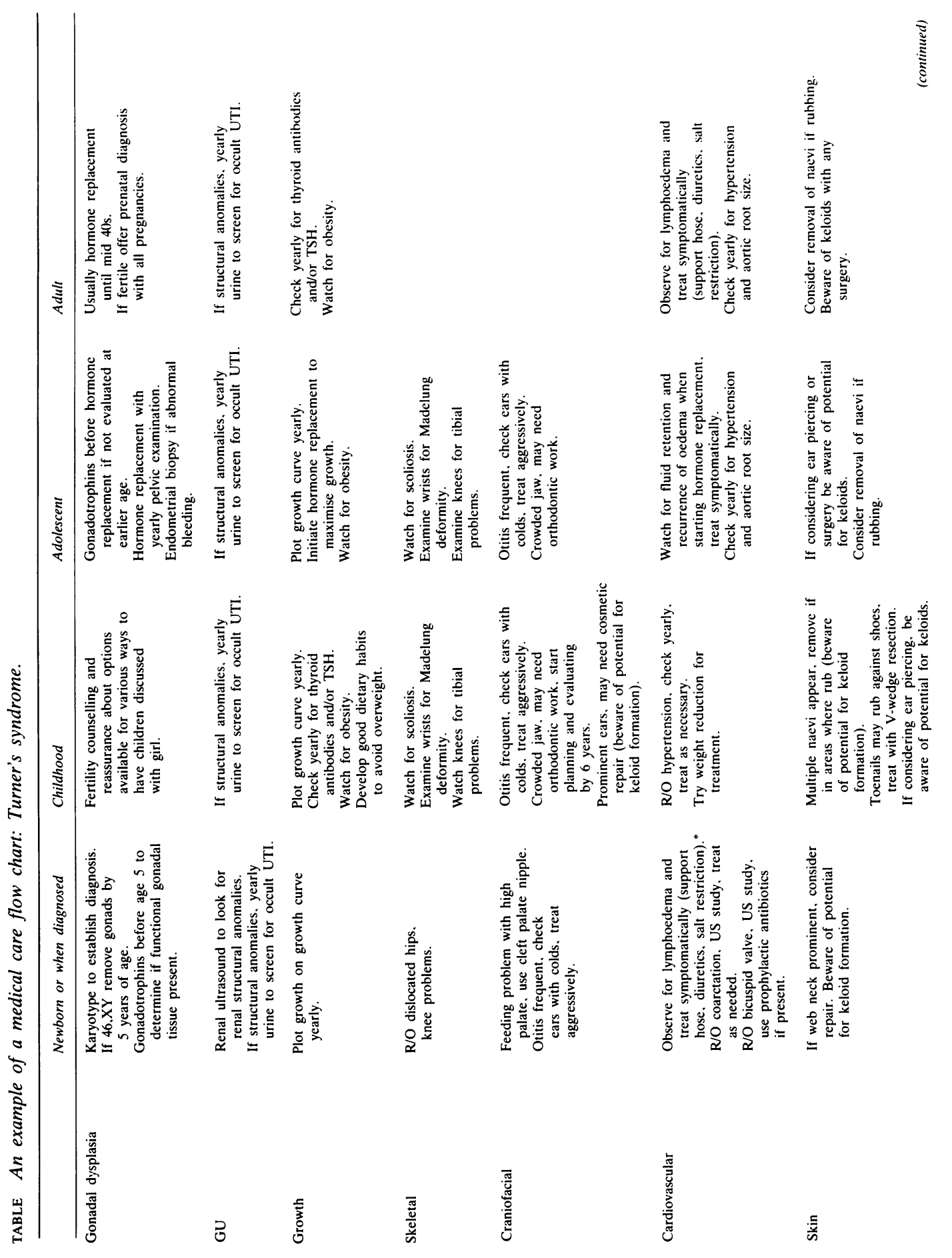

$\stackrel{\mathbb{P}}{\stackrel{9}{*}}$

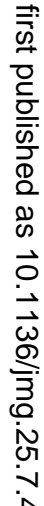

$\stackrel{\vec{\omega}}{\perp}$

은

¿

$\stackrel{\infty}{\infty}$

ญे

高

$\stackrel{2}{\rightrightarrows}$

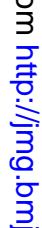

主

임

을.

N

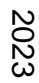

ছ

$\stackrel{0}{\stackrel{0}{\Phi}}$

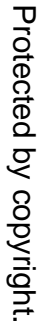




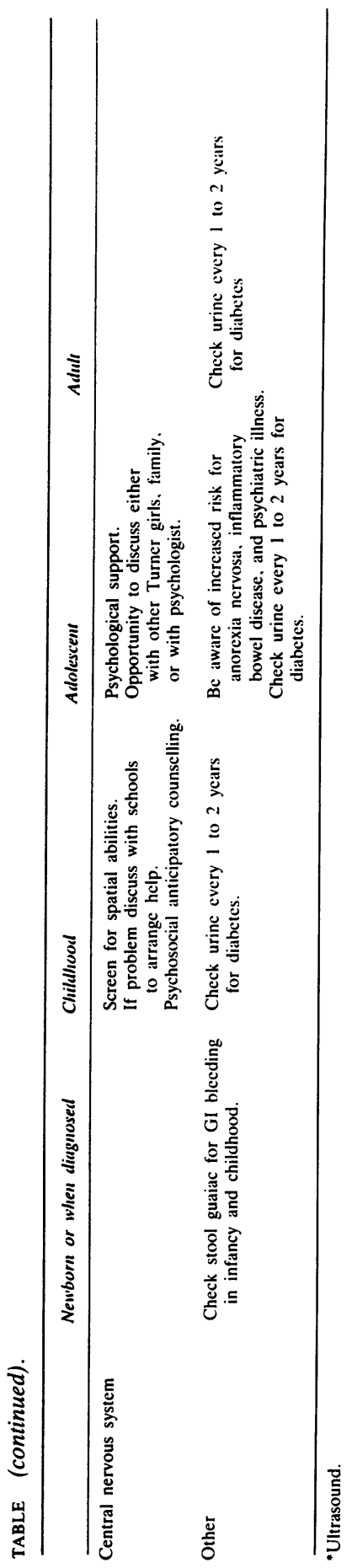

history of a disorder. For instance, it is now recognised that there is decreased incidence of arteriosclerotic disease in persons with Down's syndrome, which may be related to having three copies of the gene for homocystine synthetase (homocystinuria) which is present on chromosome $21,{ }^{47}$ as well as an increased incidence of Alzheimer's disease, which may be related to having three copies of an amyloid gene known to be on chromosome $21 .{ }^{48}$ The spectrum of causes of death in Down's syndrome has also been recently defined. ${ }^{28}$

\section{ADDITIONAL INFORMATION MUST BE COLLECTED AND A DIFFERENT TYPE OF RECORD KEPT}

In order to do studies of natural history, a specific protocol and record form need to be developed. A particular protocol is usually designed related to the known complications of the particular disorder. More information is collected than is normally part of routine medical care and long term follow up is required. A particularly careful medical workup includes family history, pregnancy history, developmental history, past medical history, review of other family records, and a thorough physical examination including detailed measurements is usually appropriate. Chronological photographs may be invaluable. There is no question but that the review and recording of this increased amount of information requires time (and hence money), must be done with great attention to detail in order to be useful, and is not part of regular health care. It is not always clear which piece of information will be important initially. The recording of natural history is an active and evolving process. Almost always, it is necessary to go through the patient record more than once when documenting the natural history, since an issue or a question will be raised by one patient which was not collected the first time in other patients.

Many groups are developing specific forms (such as the Neurofibromatosis Association) so that appropriate and detailed information can be collected. Particular areas of interest are various body measurements, changes over time, body and facial movements, various aspects of physical examination, and laboratory data, such as blood and $x$ ray studies. A form needs to be developed which is appropriate for the particular disease. It may take the form of a spread sheet, a flow chart, or a narrative. Punch cards, charts, or computers may also be used.

METHODS FOR NATURAL HISTORY STUDIES There are several methods to be considered for the collection of natural history data. These include 
cross section studies, longitudinal studies, cross grids, individual patient records, accumulated case reports, and special protocols.

\section{Cross sectional versus tongitudinal studies}

These two different methods of the study of the natural history may give quite different results, but are clearly complementary. The determination of average height, weight, and OFC by age in cross sectional studies is relatively easy to achieve if there is a large number of affected persons available. Various measurements or values from different subjects are simply tabulated. By contrast, longitudinal studies involve collecting data over time on single subjects. Longitudinal data allow individual patterns or velocities to be established, but take time and consistency to accumulate the information. When a disorder is rare, data collected over time on a single person may be the only way to establish a pattern. Through longitudinal growth studies of affected persons it may be possible to recognise different growth patterns and thereby show heterogeneity in a particular condition. ${ }^{49}$

Cross grid system involvement at various ages to demonstrate natural history

Ideally, the kinds of complications and features present at various ages and the order in which they occur can be established for a particular disorder. This documentation should reflect developmental $\stackrel{\vec{\rho}}{\vec{P}}$ and social landmarks as well as physical measure- $\overline{0}$ ment and signs at various ages. Along the axis can $\overline{0}$ be noted the body systems at risk and their potential $\frac{}{\bar{c}}$ complications. Social, psychological, developmental, $\underset{\mathbb{\mathbb { D }}}{\stackrel{2}{2}}$ and daily function skills can also be noted along the axis (figure) ${ }^{5()}$ The age is noted along the abscissa. ${ }^{\circ}$ The age of diagnosis should be noted or a line drawn $\vec{\circ}$ down the age grid. Known high risk areas should be $\overrightarrow{\vec{\omega}}$ noted as to whether they are affected or normal at a particular age.

Different disorders will involve different body areas and a broad general outline can be established $N$ that allows definition of each area. Protocols for $v$ following the potential medical complications of a $\vec{\omega}$ patient at different ages can be developed (table). $51+$ Analogy to other disorders or syndromes often gives clues to the areas that should be examined.

Individual patient's record

It is often useful for a person or a family to keep a $\overrightarrow{\mathscr{O}}$ notebook or diary of medical complications, growth, $\stackrel{.}{\circ}$ and psychological observations. Frequently, various subjective observations are clouded with time and the exact onset and sequence may become confused. Therefore, it is of great use, both in order to keep a clear record of the individual patient's medical $\frac{\mathscr{Q}}{\mathscr{Q}}$

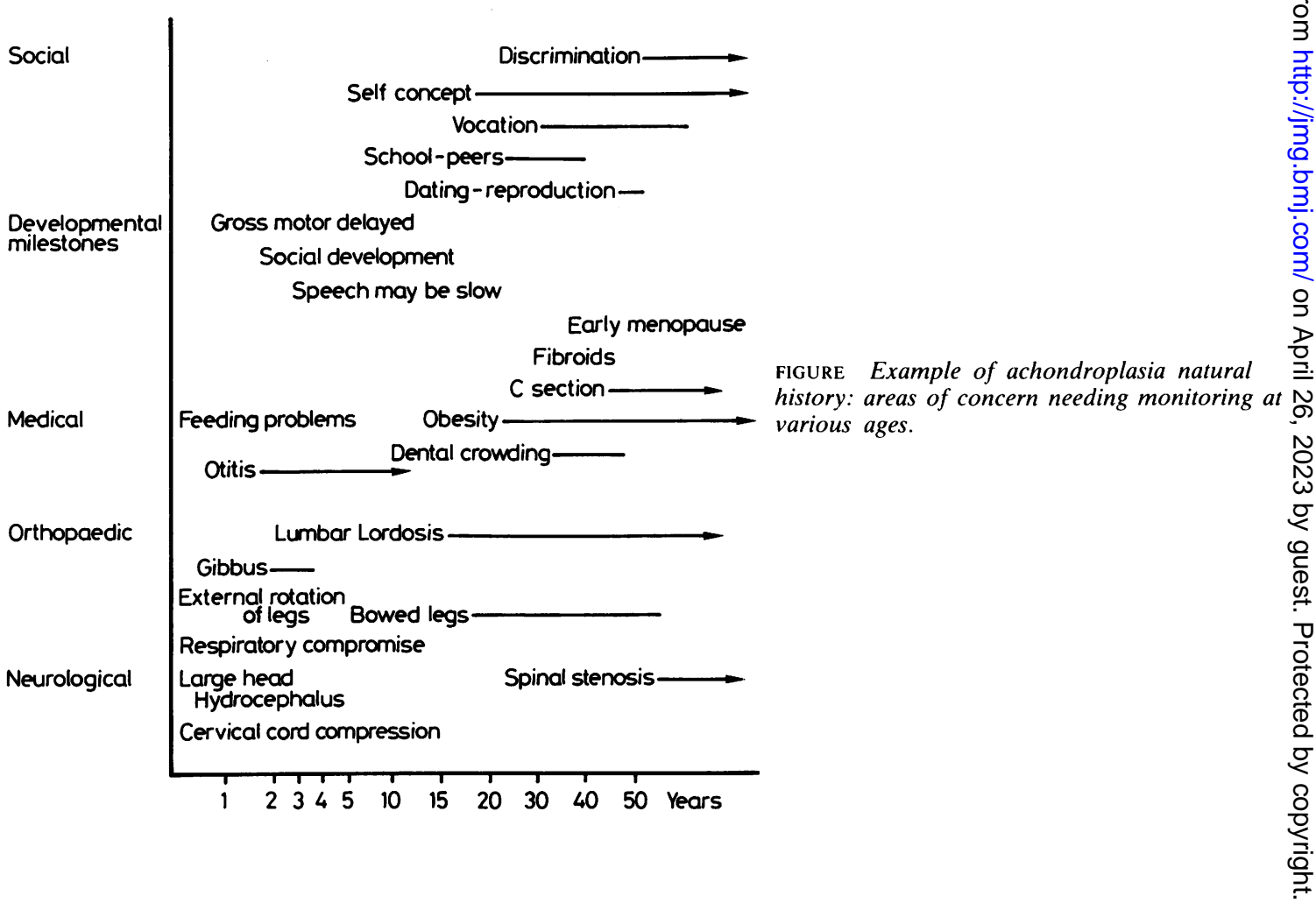


history and for the collection of natural history data, for a family with a child with a rare disorder to keep a notebook that has dates of important events, records, copies of consultations, timing of admissions to hospital, etc, so that it can be used in the future for information retrieval. Part of the notebook should include good photographs at various ages that show changes which occur over time.

\section{Individual case reports}

There is real value in single case reports of relatively rare disorders and consequently a need to continue to publish them. Unfortunately, some journals have stopped publishing them and, in the past, editors have frequently cut out some or all of the clinical data which described the natural history of a particular disorder. There are three categories of single case reports of rare disorders which are particularly useful: (1) a rare or newly recognised complication of a particular disorder, (2) a new or useful therapy for a particular disorder, and (3) a new laboratory test that better defines or identifies the disorder. Putting several single case reports together allows natural history information to emerge. Particularly unusual cases may actually provide the solution to a disorder, as did the Duchenne muscular dystrophy patient with multiple disorders who had a chromosomal deletion, ultimately leading to the isolation of the Duchenne muscular dystrophy gene. ${ }^{52}$ The patient with unusual features thus needs particularly careful evaluation.

\section{References}

1 Hall MD. Natural history of specific birth defects: introduction. Birth Defects 1977:13(3C):1-2.

2 Hall JGH. Natural history of skelctal dysplasias. In: Akeson WH. Borstein P. Glimcher MJ, eds. Symposium on heritable disorders of connective tissue. St Louis: Mosby. 1982:353-61.

3 Riccardi V. Eichner JE. Neurofibromatosis-phenotype, natural history, and pathogenesis. Baltimore: Johns Hopkins University Press, 1986.

+ US Department of Health and Human Scrvices. Neurofibromatosis. National Institutes of Health Consensus Development Conference Statement, 1.3-15 July 1987. Bethesda: US Department of Health and Human Services, 1987:6(12).

5 Allanson JE. Hall JG. Hughes HE. Preus M. Witt RD. Noonan syndrome: the changing phenotype. Am J Med Genet 1985:21: 507-14.

' Smith GF. Berg JM. Down's anomaly. 2nd ed. Edinburgh: Churchill Livingstone, 1976.

${ }^{7}$ Spranger J. Pattern recognition in bone dysplasias. In: Endocrine genetics and genetics of growth. New York: Alan R Liss. 1985:315-42.

* Wynne-Davies R. Hall CM. Apley AG. eds. Atlas of skeletal dysplasias. Edinburgh: Churchill Livingstone. 1985.

"Poznanski AK, Hernandez RJ, Guire KE, Bereza UL, Garn SM. Carpal length in children-a useful measurement in the diagnosis of rhcumatoid arthritis and some congenital malformation syndromes. Pediatr Radiol 1978:129:661-8.
1" Garn SM. Patterning in ontogeny, taxonomy, phylogeny, and dysmorphogenesis. In: Wetherington RK. ed. Colloquia in anthropology. Vol 1. Ann Arbor: The Fort Burgwin Research Center. 1977:83-106.

1 Bird TD. Ott J. Giblett ER. Chance PF. Sumi SM, Kraft GH. Genetic linkage evidence for heterogeneity in Charcot-MaricTooth neuropathy (HMSN type I). Ann Neurol 1983:14:679-84.

12 Orkin SH, Nathan DG. The molecular genetics of thalassemia. In: Harris H, Hirschhorn K. eds. Advances in human genetics. Vol 11. New York: Plenum Press. 1981.

${ }^{1.3}$ Hart KA. Hodgson S. Walker A. et al. DNA deletions in mild and severe Becker muscular dystrophy. Hum Genet 1987:75: 281-5.

It Webster's ninth new collegiate dictionary. Markham: Thomas Allen. 1986.

15 Ross R. The pathogenesis of artherosclerosis-an update. $N$ Engl J Med 1986:314:488-500.

${ }^{16}$ Mabuchi H. Sakai T. Sakai Y. et al. Reduction of serum cholesterol in heterozygous patients with familial hypercholesterolemia: additive effects of compactin and cholestyraminc. N Engl J Med 1983:308:609-13.

17 Hobbs HH. Brown MS, Russcll DW. Davignon J. Goldstein JL. Deletion in the gene for the low density lipoprotein receptor in a majority of French Canadians with familial hypercholesterolemia. $N$ Engl J Med 1987:617:734-7.

is International Nomenclature of Constitutional Diseases of Bone. Ann Radiol (Paris) 1983:26:457-62.

19) Sillence DO. Osteogenesis imperfecta: clinical variability and classification. In: Akeson WH. Bornstein P. Glimcher MJ, eds. Symposium on heritable disorders of connective tissue. St Louis: Mosby. 1982:223-37.

21) Francomano CA. Maumenec I. Liberfarb R. Pyeritz RE. Cosegregation of Stickler syndrome and type II collagen gene (COL2Al) alleles. Am J Hum Genet 1987:41:97A.

21 McKusick VA. On lumpers and splitters, or the nosology of genetic discase. Birth Defects 1969:5(1):23-30.

22 Hall JG, Reed SD, Driscoll RP. Part I. Amyoplasia: a common sporadic condition with congenital contractures. Am J Med Genet 1983;15:571-90.

23 Butler MG. Meancy FJ, Palmer CG. Clinical and eytogenetic survey of 39 individuals with Prader-Labhart-Willi syndrome. Am J Med Genet 1986:23:793-8(49.

24 Morris CA. Demsey SA, Leonard CO, Dilts C, Blackburn BL. The natural history of Williams syndrome: physical characteristics. J Pediatr 1987:113:267-73.

25 Farrer LA, Conncally PM. A genetic model for age at onset in Huntington discase. Am J Hum Genet 1985:37:350-7.

26 Horton WA. Rotter JI. Rimoin DL. Scott CI. Hall JG. Standard growth curves for achondroplasia. J Pediatr 1978:93:435-8.

27 Hecht JT. Francomano CA. Horton WA. Annegers JF. Mortality in achondroplasia. Am J Hum Genet 1987:41:454-64.

$2 \times$ Baird PA. Sadovnick AD. Life expectancy in Down syndrome. J Pediatr 1987:110:849-54.

2) Horton WA. Hall JG. Scott CI, Pyeritz RE, Rimoin DL. Growth curves for height for diastrophic dysplasia. spondylocphiphyscal dysplasia congenita. and pscudoachondroplasia. Am J Dis Child 1982:136:316-9.

3) Ranke MB. Pfluger H. Rosendahl W, et al. Turner syndrome: spontaneous growth in 150 cases and review of the literature. Eur J Pediatr 1983;141:81-8.

31 Roberts DF. Aherne GES. Retinoblastoma. In: Emery AEH. Rimoin DL, eds. Principles and practice of medical genetics. Vol 1. New York: Churchill-Livingstone, 1983:539-54.

32 Harper PS. Myotonic dystrophy and related disorders. In: Emcry AEH. Rimoin DL. eds. Principles and practice of medical genetics. Vol 1. New York: Churchill-Livingstonc. 1983:426-41.

${ }^{33}$ Lewis RA. Crowder WE, Eicrman LA, Nussbaum RL. Ferrell FE. The Gardner syndrome-significance of ocular features. Ophthalmology 1984:91:916-25. 
${ }^{34}$ Pyeritz RE, Sack GH, Udvarhelyi GB. Thoracolumbosacral laminectomy in achondroplasia: long-term results in 22 patients. Am J Med Genet 1987;28:433-44.

35 Palmer PM, MacEwen GD, Bowen JR, Mathews PA. Passive motion therapy for infants with arthrogryposis. In: Urist MR. ed. Clinical orthopedics and related research. Philadelphia: Lippencott, 1985:54-9.

${ }^{36}$ Froster-Iskenius U, Waterson J, Hall JG. A recessive form of congenital contractures and torticollis associated with malignant hyperthermia. J Med Genet 1988;25:104-12.

37 Murdoch JL, Walker BA, Hall JG, Abbey H, Smith KK, McKusick VA. Achondroplasia-a genetic and statistical survey. Ann Hum Genet 1970;33:227-44.

${ }^{38}$ Witt DR, Hayden MR, Holbrook KA, Dale BA, Baldwin VJ, Taylor GP. Restrictive dermopathy: a newly recognised autosomal recessive skin dysplasia. Am J Med Genet 1986;24:631-48.

39 Newman CGH. Clinical aspects of thalidomide embryopathy-a continuing preoccupation. In: Sever JL, Brent RL, cds. Teratogen update: environmentally induced birth defect risks. Ncw York: Alan R Liss, 1986:1-12.

4) Schutgens RBH, Heymans HSA, Wanders RJA, Bosch HVD, Tager JM. Peroxisomal disorders: a newly recognised group of genetic diseases. Eur J Pediatr 1986;144:430-40.

41 Hall JG. The analysis of Pena-Shokeir phenotypc. Am J Med Genet 1986;25:99-117.

42 Wexler NS, Young AB, Tanzi RE. Homozygotes for Huntington's disease. Nature 1987;326:194-7.

${ }^{43}$ Happle R. The McCune-Albright syndrome: a lethal gene surviving by mosaicism. Clin Genet 1986;29:321-4.

44 Phillips JA, Kazazian HH. Hacmoglobinopathics and thalassaemias. In: Emery AEH, Rimoin DL, eds. Principles and practice of medical genetics. Vol 2. Edinburgh: ChurchillLivingstone, 1983:1019-43.
45 National Clearinghouse For Human Genetic Discascs, PO Box 28612, Washington DC 20005, USA.

th Turner's Syndrome Socicty, York University, Bchavioural Science Building. Toronto, Canada.

${ }^{47}$ Chadefaux B, Rethoré MO, Raoul O, et al. Cystathionine beta synthase: gene dosage effect in trisomy 21. Biochem Biophys Res Commun 1985;128:40-4.

18 Robakis NK. Wisnicwski HM, Jenkins EC, et al. Chromosome 21q21 sublocalisation of gene encoding beta-amyloid peptide in cerebral vessels and neuritic (senilc) plaques of people with Alzheimer disease and Down syndrome. Lancet 1987;i:384-5.

${ }^{49}$ Hall JG. In utero movement and use of limbs are necessary for normal growth: a study of individuals with arthrogryposis. In: Papadatos J, Bartsocas CS, cds. Endocrine genetics and genetics of growth. New York: Alan R Liss, 1985:155-62.

5) Hall JG. Natural history of achondroplasia. Proceedings of the Ist International Conference on Human Achondroplasia, Rome, 1986.

s1 Hall JG. The management of the adult with Turner syndrome- : the natural history of Turner syndrome: In: Rosenfeld R, Grumbach M, eds. Proceedings of the International Turner Syndrome Symposium, 1987.

52 Kunkel LM, Monaco AP, Middlesworth W, Ochs HD, Latt SA Specific cloning of DNA fragments absent from the DNA of a male patient with an $\mathrm{X}$ chromosome deletion. Proc Natl Acad Sci USA 1985;82:4778-82.

Correspondence and requests for reprints to $\mathrm{Dr}$ Judith G Hall, UBC Clinical Genetics Unit, Grace Hospital, 4490 Oak Street, Vancouver, British Columbia, Canada V6H 3 V5. 